\title{
Hybrid Analytical/Numerical Solution of the Unsteady Heat Conduction Equation Subject to Unequal Robin Boundary Conditions
}

\author{
Antonio Campo \\ School of Mechanical Engineering (Escuela de Ingeniería Mecánica), Pontifical Catholic University of Valparaíso (Pontificia \\ Universidad Católica de Valparaíso), Viña del Mar, Valparaíso, Chile.
}

How to cite this paper: Antonio Campo. (2021) Hybrid Analytical/Numerical Solution of the Unsteady Heat Conduction Equation Subject to Unequal Robin Boundary Conditions. Journal of Applied Mathematics and Computation, 5(4), 303-314. DOI: 10.26855/jamc.2021.12.008

Received: September 22, 2021

Accepted: October 20, 2021

Published: November 23, 2021

*Corresponding author: Antonio Campo, School of Mechanical Engineering (Escuela de Ingeniería Mecánica), Pontifical Catholic University of Valparaíso (Pontificia Universidad Católica de Valparaíso), Viña del Mar, Valparaíso, Chile.

Email: campanto@yahoo.com

\begin{abstract}
The primary objective of the present study is to utilize the Method of Lines (MOL) for the analysis of the unsteady, heat conduction in a slab with different Robin boundary conditions. A hot fluid is in contact with the left side of the slab and a cold fluid is in contact with the right side. In the heat conduction equation, MOL discretizes the second spatial derivative while leaving the time derivative continuous. This operation leads to a system of adjoint ordinary differential equations of first order for each line in the special computational domain that is solved analytically (not numerically) with the potent eigenvalue method. The computational procedure uses a symbolic algebra code that produces the collection of eigenvalues and eigenvectors. Based on this, a sequence of piecewise temperature-time variations at each line are expressed in terms of linear combinations of exponential functions of time. A limiting test case involves a slab with asymmetric Dirichlet boundary conditions, a particular case of asymmetric Robin boundary conditions. The combination of MOL, the eigenvalue method and a symbolic algebra code delivers a set of analytical/numerical temperature-time variations exhibiting excellent quality for the entire time domain.
\end{abstract}

\section{Keywords}

Unsteady heat conduction equation, slab, unequal Robin boundary conditions, Method of Lines (MOL), eigenvalue method, symbolic algebra code, approximate analytical/numerical temperature variations

\section{Highlights}

- Unsteady, heat conduction in a slab with dissimilar Robin boundary conditions

- $\quad$ Application of the Method of Lines (MOL) to the unsteady heat conduction equation

- The eigenvalue method solves the adjoint system of ordinary differential equations of first order

- The temperature-time variations are expressed in terms of linear combinations of exponential functions of time

\section{Introduction}

If the unsteady heat conduction equation is linear and the boundary conditions in the domain are homogeneous, the method of separation of variables is a popular exact, analytical avenue as cited in textbooks on heat conduction [1-8]. The rationale is that the boundary value problem via an ordinary differential equation of second order in the space variable 
accommodates to the Sturm-Liouville problem. Without this unique comportment, the problem would not be an eigenvalue problem at all, and therefore it is unlikely that enough solutions could be found to solve the unsteady heat conduction equation.

A review of the literature reveals that the problem of unsteady heat conduction in a slab involving constant initial temperature and unequal temperature boundary conditions has been treated with the Numerical Method of Lines (NMOL) in Reference [9]. The corresponding system of first order, linear ordinary differential equations was solved numerically with the Runge-Kutta method of fourth/fifth order using the MATLAB program ode45. In fact, the Dirichlet boundary condition is the easiest boundary condition [1-8].

The primary objective of the present study to implement the Method of Lines (MOL), not the Numerical Method of Lines (NMOL) for the analysis of the unsteady heat conduction equation in a slab with unequal Robin boundary conditions imposed at the surfaces. The adjoint system of linear, first order ordinary differential equations is readily solved analytically (not numerically) with the potent eigenvalue method.

\section{Physical System and Mathematical Formulation}

Consider unsteady heat conduction in the large slab of thickness $L$ sketched in Figure 1 . The thermal conductivity $k$ and thermal diffusivity $\alpha$ of the solid are assumed constant or nearly constant. The initial temperature of the slab is uniform and equal to $T_{i n}$. For time $t>0$, the left surface of the slab is exposed to a hot fluid 1 at temperature $T_{f 1}$ with convection coefficient $h_{1}$, whereas the right surface is exposed to a cold fluid 2 at temperature $T_{f 2}$ with convection coefficient $h_{2}$. According to the description, the unsteady heat conduction equation is

$$
\frac{\partial T}{\partial t}=\alpha \frac{\partial^{2} T}{\partial x^{2}} \quad \text { in } 0<x<L
$$

The initial condition is

$$
T=T_{\text {in }}, \quad t=0
$$

and the boundary conditions are

$$
\begin{array}{rrr}
-k \frac{\partial T}{\partial x}=h_{1}\left(T_{f 1}-T\right), & x=0 & \text { for convection heating } \\
-k \frac{\partial T}{\partial x}=h_{2}\left(T-T_{f 2}\right), & x=L & \text { for convection cooling }
\end{array}
$$

In the classification of boundary conditions, eqs. (3a) and (3b) are classified as Robin boundary conditions (Özişik [5]) or boundary condition of third kind (Luikov [2]). Physically, the Robin boundary condition represents a thermodynamic energy balance at the solid/fluid interface between heat conduction in a solid and heat convection in a fluid. Correspondingly, it is also called convective boundary condition (Arpaci [1]).

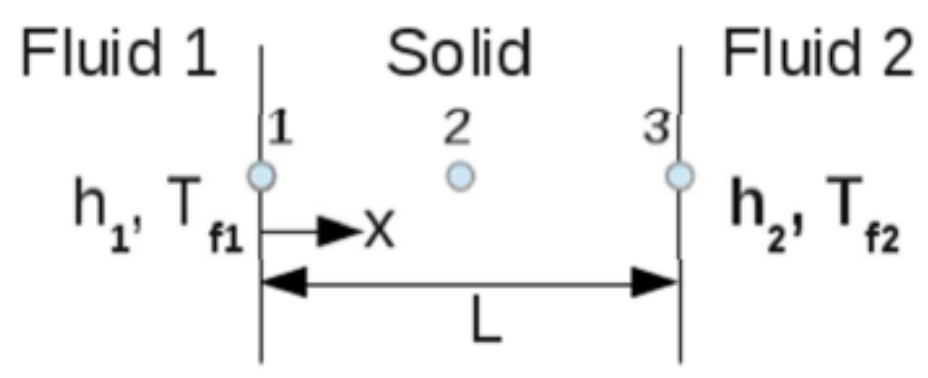

Figure 1. Special computational domain containing three lines, $\Delta x=\frac{L}{2}$.

\subsection{Particular case of importance}

A particular case of unsteady heat conduction in a slab deals with vigorous convection heating on the left surface $x=0$ in the slab united to vigorous convection cooling on the right surface $x=L$. Under these circumstances, eqs. (3a) and (3b) are gradually transformed into 


$$
\begin{array}{llll}
T=T_{f 1}, & x=0 & \text { when } & h_{1} \rightarrow \infty \\
T=T_{f 2}, & x=L & \text { when } & h_{2} \rightarrow \infty
\end{array}
$$

which are unequal Dirichlet boundary conditions.

\section{Computational Procedure}

If the unsteady heat conduction equation is non-homogeneous and/or the boundary conditions are non-homogeneous, the principle of superposition of solutions is a reasonable computational procedure to be employed (Arpaci [1]), Özişik [5], Poulikakos [6] and Mackowski [9]. Because any superposition of solutions of a linear, homogeneous partial differential equation is again a solution, then the particular solutions may then be combined to obtain general solutions. For instance, if $u_{1}$ and $u_{2}$ are solutions of a partial differential equation in the region $R$, then $u=C_{1} u_{1}+C_{2} u_{2}$ with any constants $C_{1}$ and $C_{2}$, is also a solution of the partial differential equation in the region $R$. In this category, the decomposition begins by splitting the original unsteady one-dimensional heat conduction problem into two sub-problems, one sub-problem contains an unsteady part and the other sub-problems contain a steady part, which includes the non-homogeneous boundary conditions. The unsteady part will conform to the requirements of the method of separation of variables, i.e., the unsteady part has homogeneous boundary conditions and an inhomogeneous initial condition, which is the form that can be tackled by the method of separation of variables. The drawback posed by the principle of superposition of solutions is that sometimes the final solution is a fairly complicated mosaic of individual solutions. Owing to the ill-behavior, the principle of superposition turns out to be strenuous.

Additionally, the method of variation of parameters is considered a general method for solving an inhomogeneous unsteady heat conduction and/or inhomogeneous boundary conditions (Poulikakos [6]). In principle, the method of variation of parameters consists on a single series expansion of eigenfunctions. Usually, the method of variation of parameters is more involved than the principle of superposition.

In view of the foregoing, it is clear that both the principle of superposition of solutions and the method of variation of parameters constitute complex computational procedures and of course it is logical to explore simpler approaches. A methodology that has not been reported in the archival literature for solving the unsteady heat conduction equation subject to a variety of boundary conditions of non-homogeneous type is the powerful Method of Lines (MOL).

The primary objective of the present study is to determine the asymmetrical temperature field $T(x, t)$ in the slab affected by unequal Robin boundary conditions accounting for different convection coefficients and different fluid temperatures. To accomplish the task, an approximate hybrid analytical/numerical method called the Method of Lines (MOL) will be investigated. MOL is expounded in the following section.

\section{Synopsis of the Method of Lines (MOL)}

In general, the Method of Lines (MOL) embodies a hybrid analytical/numerical computational procedure for treating partial differential equations of parabolic type in semi analytical manner (Liskovets [10]). The basic idea behind MOL is to discretize the spatial derivatives in the PDE with algebraic approximations, while the time derivative remains continuous. This operation transforms the PDE into an adjoint system of ordinary differential equations of first order. As a direct result, the solution of the transformed system of ODEs seeks to approximate the solution of the original PDE. From an applied mathematics perspective, the primary advantage inherent to MOL is that an adjoint system of ordinary differential equations is easier to solve than the original partial differential equation. In general, the required algebraic approximation of the spatial derivatives in the PDE can be based on finite differences, finite elements, collocations, etc. Obviously, the method of finite differences being the easiest algebraic approximation will be employed in this study.

In the unsteady heat conduction equation, the independent variables are the space coordinate $x$ and time $t$, MOL discretizes the spatial derivative approximating the PDE by a system of first-order ordinary differential equations in $t$. Therefore, within the platform of solutions, the Method of Lines (MOL) replaces the exact, analytical solution $u(x, t)$ at an infinite number of points in the space-time domain with an approximate semi-analytical solution $u_{j}(t), j=1,2, \ldots N$ on a finite number of lines displayed in the space-time domain. In order to apply MOL to eq. (1), a special computational domain needs to be constructed with a group of straight lines perpendicular to the $x$-coordinate. For convenience, the straight lines are separated by equal spatial intervals $\Delta x=x_{j+1}-x_{j}$.

In eq. (1), the second order spatial derivative $\frac{\partial^{2} T}{\partial x^{2}}$ is discretized with the three-point central finite-difference approximation 


$$
\left.\frac{\partial^{2} T}{\partial x^{2}}\right|_{x_{j}} \approx \frac{T_{j-1}-2 T_{j}+T_{j+1}}{(\Delta x)^{2}}+O(\Delta x)^{2}
$$

where the appended term $O(\Delta x)^{2}$ stands for the truncation error of second order (Isaacson and Keller [11])

Inserting eq. (4) into eq. (1) creates the following adjoint system of ordinary differential equations of first order in $t$,

$$
\frac{d T_{j}}{d t}=\frac{\alpha}{(\Delta x)^{2}}\left(T_{j-1}-2 T_{j}+T_{j+1}\right)+O(\Delta x)^{2}, \quad j=1,2, \ldots, M
$$

along with the discretized initial conditions

$$
T_{j}=T_{i n}, t=0, \quad j=1,2, \ldots, M
$$

For closure, the adjoint system of $M$ ordinary differential equations of first order in eq. (5) must absorb the two boundary conditions stated in eqs. (3a) and (3b).

An important advantage possessed by MOL is that the errors are quantified in terms of the spatial interval $\Delta x$ solely because time is continuous, i.e., there is no time interval $\Delta t$ [11].

From a fundamental framework, the original initial boundary value problem (IBVP) given by the set of eqs. (1), (2), (3a) and (3b) is replaced by an initial value problem (IVP) given by the pair of eqs. (5) and (6). An important advantage possessed by MOL is that the errors are quantified in terms of the spatial interval $\Delta x$ solely because time is continuous, i.e., there is no time interval $\Delta t[11]$.

The approximate MOL solution $T_{j}(t), j=1,2, \ldots M$ can be obtained either analytically or numerically. Obviously, analytical solutions are preferable because they are very elegant, more accurate and faster. In fact, the main advantage of an analytical solution over a numerical solution is its closed-form structure (instead of a long list of numbers), allowing the analyst to gain valuable physical insight. In this context, the eigenvalue method (Golub and van der Vorst [12]) is viewed as a robust method to solve analytically the adjoint system of ordinary differential equations of first order in eq. (5), which can be rewritten in vector form as

$$
x^{\prime}(t)=\boldsymbol{A} x(t)
$$

where $\boldsymbol{A}$ is a matrix of coefficients. Thereafter, the exact, analytical solution of eq. (7) is of the form

$$
x(t)=v \exp (\lambda t)
$$

where $\lambda$ is an eigenvalue and $\boldsymbol{v}$ is an associate eigenvector.

In principle, when the limit of the RHS in eq. (5) is taken as the spatial interval $\Delta x \rightarrow 0$, the adjoint system of ordinary differential-algebraic equations of first order collapses with the original parabolic partial differential equation (1), so that the two solutions are identical.

Important issues related to the Method of Lines (MOL) applied to time-dependent partial differential equations of parabolic type have been addressed for convergence by Verwer and Sanz-Serna [13], for stability by Reddy and Trefethen [14] and for errors by Zafarullah [15].

As additional information, the Method of Lines (MOL) and its variants the Numerical Method of Lines (NMOL), the Transversal Method of Lines (MOL) and the Improved Transversal Method of Lines (MOL) have been reported by Campo and collaborators (see References [16-21].

\section{Pursuit of the Method of Lines (MOL)}

\section{Case 1: Three active lines}

As the first attempt, we choose the coarsest spatial interval $\Delta x=\frac{L}{2}$ in eq. (5) creating three active lines: line 1, line 2 and line 3 as shown Figure 1. In fact, the crude line arrangement is the minimum that could be done within the horizon of MOL. Thereby, the resulting adjoint system of three coupled ordinary differential equations of first-order is

$$
\begin{aligned}
& \frac{d T_{1}}{d t}=\frac{\alpha}{(\Delta x)^{2}}\left(T_{0}-2 T_{1}+T_{2}\right) \\
& \frac{d T_{2}}{d t}=\frac{\alpha}{(\Delta x)^{2}}\left(T_{1}-2 T_{2}+T_{3}\right)
\end{aligned}
$$




$$
\frac{d T_{3}}{d t}=\frac{\alpha}{(\Delta x)^{2}}\left(T_{2}-2 T_{3}+T_{4}\right)
$$

Next, the two boundary conditions stated in eq. (3a) and (3b) are incorporated.

To deal with enhanced accuracy, the first order spatial derivative $\frac{\partial T}{\partial x}$ in eq. (3a) is handled with the two-point central formulation having second order error $O(\Delta x)^{2}$ (Isaacson and Keller [11])

$$
\left.\frac{\partial T}{\partial x}\right|_{x_{j}} \approx \frac{T_{j+1}-T_{j-1}}{2 \Delta x}+O(\Delta x)^{2}
$$

Then, the resulting algebraic equation is

$$
-k\left(\frac{T_{2}-T_{0}}{2 \Delta x}\right)=h_{1}\left(T_{f 1}-T_{1}\right)
$$

From here, the temperature $T_{0}$ lying at the fictitious line 0 outside of the special computation domain in Figure 1 is expressed as

$$
T_{0}=T_{2}+\frac{2 \Delta x h_{1}}{k}\left(T_{f 1}-T_{1}\right)
$$

Substituting $T_{0}$ into eq. (9) delivers the following ordinary differential equation of first-order

$$
\frac{d T_{1}}{d t}=\frac{\alpha}{(\Delta x)^{2}}\left[2 T_{2}-\left(\frac{2 \Delta x h_{1}}{k}+2\right) T_{1}+\left(\frac{2 \Delta x h_{1}}{k}\right) T_{f 1}\right]
$$

Likewise, the same two-point central formulation with second order error $O(\Delta x)^{2}$ stated in eq. (12) provides the algebraic equation

$$
-k\left(\frac{T_{4}-T_{2}}{2 \Delta x}\right)=h_{2}\left(T_{3}-T_{f 2}\right)
$$
by

Hence, the temperature $T_{4}$ lying at the fictitious line 4 outside of the computational domain in Figure 1 is expressed

$$
T_{4}=T_{2}-\frac{2 \Delta x h_{2}}{k}\left(T_{3}-T_{f 2}\right)
$$

Substituting $T_{4}$ into eq. (11) results in the following ordinary differential equations of first-order

$$
\frac{d T_{3}}{d t}=\frac{\alpha}{(\Delta x)^{2}}\left[2 T_{2}-\left(\frac{2 \Delta x h_{2}}{k}+2\right) T_{3}+\left(\frac{2 \Delta x h_{2}}{k}\right) T_{f 2}\right]
$$

Consequently, the coupled system of three ordinary differential equations of first-order consists in eqs. (15), (10) and (18). Further, the initial conditions in eq. (6) are converted to

$$
T_{1}=T_{2}=T_{3}=T_{i n}, t=0
$$

\section{Case 2: Three active lines and two inactive lines}

The spatial interval is halved to $\Delta x=\frac{L}{4}$, generating five lines: line 1, line 2, line 3, line 4 and line 5 as shown in Figure 2. Specifically, the three interior lines: line 2, line 3 and line 4 establish three active lines, whereas the two boundary lines: lines 1 and 4 are inactive lines. Correspondingly, the system of three coupled ordinary differential equations of first order is 


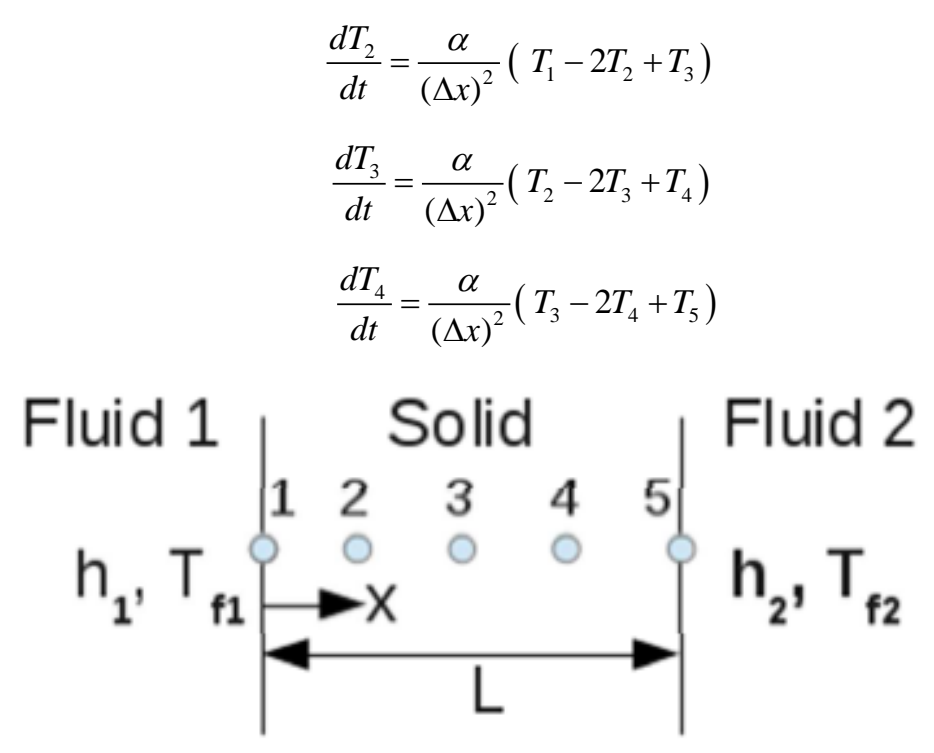

Figure 2. Special computational domain containing five lines, $\Delta x=\frac{L}{4}$.

The piecewise initial conditions from eq. (6) are

$$
T_{2}=T_{3}=T_{4}=T_{\text {in }}, \quad t=0
$$

For enhanced accuracy, the first order spatial derivative $\frac{\partial T}{\partial x}$ in eq. (3a) is replaced by the three-point forward formulation with second order error $O(\Delta x)^{2}$ [11]:

$$
\left.\frac{\partial T}{\partial x}\right|_{x_{j}} \approx \frac{-T_{j+2}+4 T_{j+1}-3 T_{j}}{2 \Delta x}+O(\Delta x)^{2}
$$

Thereby, the algebraic approximation equivalent to eq. (3a) corresponds to

$$
-k\left(\frac{-T_{3}+4 T_{2}-3 T_{1}}{2 \Delta x}\right)=h_{1}\left(T_{f 1}-T_{1}\right)
$$

Consequently, the temperature $T_{1}$ at the inactive line 1 is evaluated with the companion algebraic equation

$$
T_{1}=\frac{1}{\left(\frac{2 \Delta x h_{1}}{k}+3\right)}\left[-T_{3}+4 T_{2}+\left(\frac{2 \Delta x h_{1}}{k}\right) T_{f 1}\right]
$$

Similarly, he replacement of the first spatial derivative $\frac{\partial T}{\partial x}$ with the three-point backward formulation having second order error $O(\Delta x)^{2}$ [11] in eq. (3b) is

$$
\left.\frac{\partial T}{\partial x}\right|_{x_{j}} \approx \frac{3 T_{j+2}-4 T_{j+1}+T_{j}}{(2 \Delta x)}+(\Delta x)^{2}
$$

Hence, this option provides the algebraic approximation

$$
-k\left(\frac{3 T_{5}-4 T_{4}+T_{3}}{2 \Delta x}\right)=h_{2}\left(T_{5}-T_{f 2}\right)
$$


which is equivalent to eq. (3b). Finally, the temperature $T_{5}$ at the inactive line 5 is evaluated from the companion algebraic equation

$$
T_{5}=\frac{1}{\left(\frac{2 \Delta x h_{2}}{k}+3\right)}\left[-T_{3}+4 T_{4}+\left(\frac{2 \Delta x h_{2}}{k}\right) T_{f 2}\right]
$$

In retrospect, Case 2 engages two different types of equations:

1) a system of three coupled ordinary differential equations of first-order for temperatures $T_{2}, T_{3}, T_{4}$ at the active lines 2, 3, 4 written in eqs. (17)-(19), subject to the three initial conditions stated in eq. (23).

2) two companion algebraic equations for temperatures $T_{1}, T_{5}$ at the inactive lines 1,5 written in eqs. (26) and (29).

\section{Case 3: Five active lines}

The spatial interval is halved to $\Delta x=\frac{L}{4}$ so that all lines: line 1, line 2, line 3, line 4 and line 5 to be active as shown in Figure 2. Accordingly, the system of five ordinary differential equations of first order is comprised of

$$
\begin{gathered}
\frac{d T_{1}}{d t}=\frac{\alpha}{(\Delta x)^{2}}\left(T_{0}-2 T_{1}+T_{2}\right) \\
\frac{d T_{2}}{d t}=\frac{\alpha}{(\Delta x)^{2}}\left(T_{1}-2 T_{2}+T_{3}\right) \\
\frac{d T_{3}}{d t}=\frac{\alpha}{(\Delta x)^{2}}\left(T_{2}-2 T_{3}+T_{4}\right) \\
\frac{d T_{4}}{d t}=\frac{\alpha}{(\Delta x)^{2}}\left(T_{3}-2 T_{4}+T_{5}\right) \\
\frac{d T_{5}}{d t}=\frac{\alpha}{(\Delta x)^{2}}\left(T_{4}-2 T_{5}+T_{6}\right)
\end{gathered}
$$

For enhanced accuracy, the first spatial derivative $\frac{\partial T}{\partial x}$ in eq. (3a) is treated with the two-point central formulation having second order error $O(\Delta x)^{2}$ stated in eq. (12). In turn, this step gives rise to the companion algebraic approximation

$$
-k\left(\frac{T_{2}-T_{0}}{2 \Delta x}\right)=h_{1}\left(T_{f 1}-T_{1}\right)
$$

In here, the temperature $T_{0}$ sits on the fictitious line 0 that lies outside the special computational domain shown in Figure 2. $T_{0}$ is evaluated with the algebraic equation

$$
T_{0}=T_{2}+\frac{2 \Delta x h_{1}}{k}\left(T_{f 1}-T_{1}\right)
$$

Substituting eq. (36) into eq. (30) eliminates $T_{0}$. and leads to the ordinary differential equations of first order

$$
\frac{d T_{1}}{d t}=\frac{\alpha}{(\Delta x)^{2}}\left[2 T_{2}-\left(\frac{2 \Delta x h_{1}}{k}+2\right) T_{1}+\left(\frac{2 \Delta x h_{1}}{k}\right) T_{f 1}\right]
$$

Similarly, applying the two-point central formulation to the first spatial derivative $\frac{\partial T}{\partial x}$ with second order error $O(\Delta x)^{2}$ in eq. (3b) produces the algebraic approximation 


$$
-k\left(\frac{T_{6}-T_{4}}{2 \Delta x}\right)=h_{2}\left(T_{5}-T_{f 2}\right)
$$

Thereupon, the temperature $T_{6}$ sits on the fictitious line 6 that lies outside the special computational domain in Figure 2 and is evaluated with the companion algebraic equation

$$
T_{6}=T_{4}-\frac{2 \Delta x h_{2}}{k}\left(T_{5}-T_{f 2}\right)
$$

Substituting eq. (39) into eq. (33) eliminates $T_{6}$. Hence, this ends up in the ordinary differential equations of first order

$$
\frac{d T_{5}}{d t}=\frac{\alpha}{(\Delta x)^{2}}\left[2 T_{4}-\left(\frac{2 \Delta x h_{2}}{k}+2\right) T_{5}+\left(\frac{2 \Delta x h_{2}}{k}\right) T_{f 2}\right]
$$

Consequently, Case 3 accounts for the system of five coupled ordinary differential equations of first-order given by eqs. (36), (31), (32), (33) and (40), which is subject to five initial conditions

$$
T_{1}=T_{2}=T_{3}=T_{4}=T_{5}=T_{i n}, t=0
$$

Any of three symbolic software codes Mathematica, Maple and Matlab cited in [12] can be utilized to solve analytically the three systems of coupled ordinary differential equations of first order associated with Cases 1,2 and 3 under study. In the three cases, the eigenvalue method demonstrated to be a formidable procedure for computing the sequence of eigenvalues $\lambda$ and associate eigenvectors $v$ in the symbolic software environment.

\section{Presentation of Temperature Results}

The computed dimensionless temperatures will be presented for the particular case engaging vigorous convection heating of the slab with a hot fluid temperature $T_{f 1}$ and high convection coefficient $h_{1}$ on the left surface of the slab in conjunction with vigorous convection cooling of the slab with a cold fluid temperature $T_{f 2}$ and low convection coefficient $h_{2}$ on the right surface.

For convenience, the following dimensionless variables for temperature $T$, space variable $x$ and time $t$ are adopted:

$$
\theta=\frac{T-T_{f 2}}{T_{f 1}-T_{f 2}}, \quad X=\frac{x}{L}, \quad \tau=\frac{t}{L^{2} / \alpha}
$$

The dimensionless version of eq. (1) is

$$
\frac{\partial \theta}{\partial \tau}=\frac{\partial^{2} \theta}{\partial X^{2}} \quad \text { in } 0<\quad X<1
$$

The initial condition is

$$
\theta=1, \tau=0
$$

and the boundary conditions are

$$
\begin{array}{ll}
\theta=1, & X=0 \\
\theta=0, & X=1
\end{array}
$$

The dimensionless space interval $\Delta X=\frac{1}{4}$ used in Case 3 is assigned to eq. (5). That is

$$
\frac{d \theta_{j}}{d \tau}=\frac{\theta_{j-1}-2 \theta_{j}+\theta_{j+1}}{(1 / 4)^{2}}, \quad j=1,2, \ldots, M
$$

This equation is expected to have a truncation error of $O\left(\frac{1}{4}\right)^{2}$. Applying the boundary conditions stated in eqs. (45a) and (45b) supplies the following system of coupled ordinary differential equations of first order and non homogeneous

$$
\begin{gathered}
\frac{d \theta_{1}}{d \tau}=16 \theta_{2}-32 \theta_{1}+16 \\
\frac{d \theta_{2}}{d \tau}=16 \theta_{3}-32 \theta_{2}+16 \theta_{1} \\
\frac{d \theta_{3}}{d \tau}=-32 \theta_{3}+16 \theta_{2}
\end{gathered}
$$


From eq. (44), proper the initial conditions are

$$
\theta_{1}=\theta_{2}=\theta_{3}=1 \quad \text { at } \quad \tau=0
$$

\section{Piecewise dimensionless temperature field}

The solution of eqs. (47) to (50) can be determined in exact, analytical form utilizing any algebraic symbolic code, like Mathematica, Maple MATLAB cited in [12]. The output delivers the piecewise dimensionless temperature field

$$
\begin{gathered}
\theta_{1}(\tau)=\frac{(2-\sqrt{2})+(2+\sqrt{2}) e^{32 \sqrt{2} \tau}+2 e^{16 \sqrt{2} \tau}\left(3 e^{32 \tau}-1\right)}{8 e^{16(2+\sqrt{2}) \tau}} \quad \text { at } X=\frac{1}{4} \\
\theta_{2}(\tau)=\frac{1}{2}+\frac{\cosh (16 \sqrt{2} \tau)+\sqrt{2} \sinh (16 \sqrt{2} \tau)}{2 e^{32 \tau}} \quad \text { at } X=\frac{1}{2} \\
\theta_{3}(\tau)=\frac{(2-\sqrt{2})+(2+\sqrt{2}) e^{32 \sqrt{2} \tau}+2 e^{16 \sqrt{2} \tau}\left(e^{32 \tau}+1\right)}{8 e^{16(2+\sqrt{2}) \tau}} \quad \text { at } X=\frac{3}{4}
\end{gathered}
$$

Notice that eqs. 51) and (53) are a finite four term series of exponential functions, whereas eq. (52) is a finite three term series of exponential functions. Evaluating eqs. (51) to (53) at $\tau=0$ the initial conditions

$$
\theta_{1}(0)=\theta_{2}(0)=\theta_{3}(0)=1
$$

are fully recovered.

Additionally, the dimensionless mean temperature field is

$$
\theta_{m}(\tau)=\int_{0}^{1} \theta(X, \tau) d X
$$

where

$$
\theta_{m}=\frac{T_{m}(t)-T_{f 2}}{T_{f 1}-T_{f 2}}
$$

comes from eq. (42).

Owing that eqs. (51), (52) and (53) are discrete in $X$, eq. (54) must be integrated numerically. Thereby, the composite $1 / 3$ Simpson rule provides the equation

$$
\theta_{m}(\tau)=\frac{1}{12}+\frac{\theta_{1}(\tau)}{3}+\frac{\theta_{2}(\tau)}{6}+\frac{\theta_{3}(\tau)}{3}
$$

Introducing eqs. (51), (52) and (53) into eq. (56) and doing the required algebra ends up with the equation for the dimensionless mean temperature field

$$
\theta_{m}(\tau)=\frac{1}{2}+\frac{5 \cosh (16 \sqrt{2} \tau)}{12 \mathbb{e}^{32 \tau}}+\frac{\sqrt{2} \sinh (16 \sqrt{2} \tau)}{4 \mathbb{e}^{32 \tau}}
$$

That is, a finite three term series involving ratios of hyperbolic functions of $\tau$ and exponential functions of $\tau$.

The values of the dimensionless approximate mean temperatures evaluated with eq. (57) along with the values of the dimensionless exact mean temperatures evaluated with eq. (A.3) are listed in Table 1 and concurrently graphed in Figure 3 for dimensionless time $\tau \geq 0.001$. It is observable in the table and figure that the relative error at a very small dimensionless time $\tau=0.001$ is $5.5 \%$. In fact, this number is compatible with the truncation error of $O\left(\frac{1}{4}\right)^{2}$, equivalent to a $6.25 \%$ relative error. Furthermore, the relative error diminishes to $2 \%$ at a small dimensionless time $\tau=0.01$ and the relative error diminishes further to $0.05 \%$ at a moderate dimensionless time $\tau=0.1$.

Table 1. Dimensionless mean temperatures

\begin{tabular}{ccccc}
\hline $\begin{array}{c}\text { Dimensionless } \\
\text { time } \tau\end{array}$ & $\begin{array}{c}\text { Dimensionless, approximate } \\
\text { mean temperature } \theta_{m, a} \\
\text { with MOL 3L* }\end{array}$ & $\begin{array}{c}\text { Dimensionless, approximate } \\
\text { mean temperature } \theta_{m, a} \\
\text { with MOL 4L* }\end{array}$ & $\begin{array}{c}\text { Dimensionless, exact } \\
\text { mean temperature } \\
\theta_{m, e} \\
\text { Özişik [5] }\end{array}$ & $\begin{array}{c}\text { Relative errors } \\
\text { in \% for MOL } \\
3 L^{*}, \text { MOL 4L* }\end{array}$ \\
\hline 0.001 & 0.9114 & 0.9276 & 0.9643 & $-5.486,-3.806$ \\
0.01 & 0.8689 & 0.8780 & 0.8872 & $-2.063,-1.037$ \\
0.1 & 0.6510 & 0.6511 & 0.6508 & $-0.046,-0.015$ \\
1 & 0.5 & 0.5 & 0.5 & 0 \\
\hline
\end{tabular}

* MOL 3L and MOL 4L refer to MOL with 3 lines and MOL with 4 lines. 


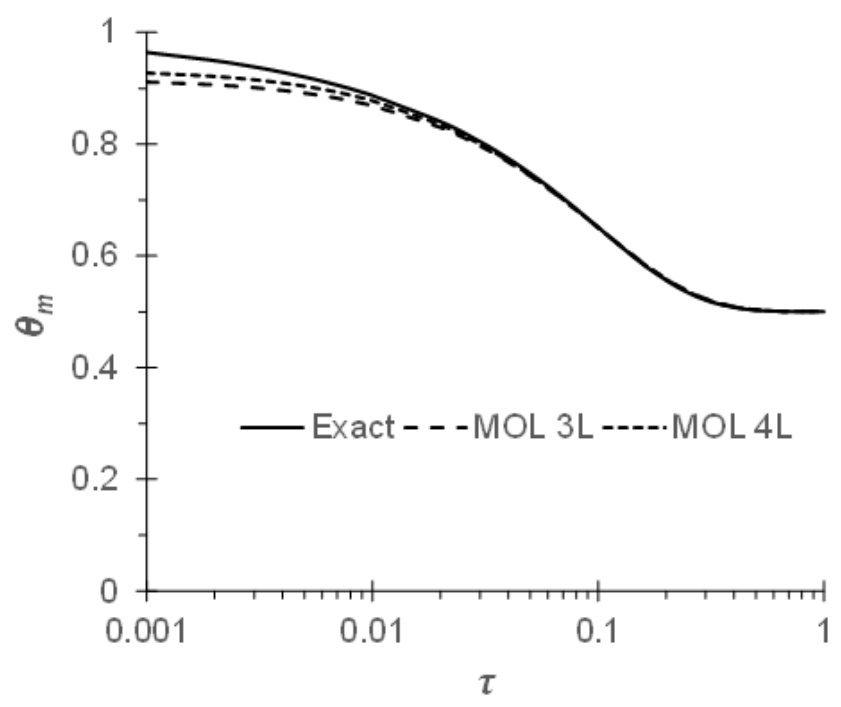

Figure 3. Graphical display of the exact and approximate mean temperature fields in dimensionless form. In the leyend, $3 \mathrm{~L}$ and 4L refer to MOL with 3 lines and MOL with 4 lines.

Needless to say, in case that dimensionless temperatures are needed at very small dimensionless time $\tau<0.001$, there are two possible avenues that could be pursued. One posible avenue is to refine the computational domain adding more lines. As a reference, the dimensionless mean temperature field with 4 lines in the special computational domain is

$$
\theta_{m}(\tau)=\frac{1}{2}+\frac{125 \cosh (12.5 \sqrt{5} \tau)+45 \sqrt{5} \sinh (16 \sqrt{5} \tau)}{288 e^{37.5 \tau}}
$$

At a very small dimensionless time $\tau=0.001$, the relative error for $\theta_{m}(\tau)$ connected to MOL with 4 lines is 3.8\%. In fact, this number is compatible with the truncation error of $O\left(\frac{1}{5}\right)^{2}$, which is equivalent to $4.0 \%$.

\section{Total heat transfer}

The total heat transfer $Q$ that takes place from/to a slab between the initial time $t=0$ and a certain final time $t_{\text {fin }}$ may be computed with heat conduction theory [1-8] or thermodynamics concepts. Opting for the easiness of the latter, the relation for the total heat transfer $Q$ is

$$
Q(t)=m c_{v}\left[T_{i n}-T_{m}(t)\right]
$$

Combining eqs. (54) and (57) results in the working equation

$$
Q(\tau)=m c_{v}\left[T_{i n}-\Delta T_{f} \theta_{m}(\tau)-T_{f 2}\right]
$$

where $\Delta T_{f}=T_{f 1}-T_{f 2}$ and $\theta_{m}(\tau)$ is given by eq. (58).

\section{Conclusions}

The main conclusion that may be drawn from the theoretical study dealing with the unsteady heat conduction in a slab subject to different Robin boundary conditions (one for the hot solid/fluid interface and the other for the cold solid/fluid interface) is that the combination of the Method of Lines (MOL), the eigenvalue method using a symbolic algebra code institutes a formidable computational procedure for determining semi-analytical/numerical temperature-time fields in the slab under study with outstanding accuracy.

\section{References}

[1] V. Arpaci. (1966). Conduction Heat Transfer. Addison-Wesley, Reading, MA, 1966.

[2] A. V. Luikov. (1968). Analytical Heat Diffusion Theory, Academic Press, London, England, UK, 1968.

[3] G. E. Myers. (1971). Analytical Methods in Conduction Heat Transfer, McGraw-Hill, New York, 1971. 
[4] U. Grigull and H. Sanders. (1984). Heat Conduction, Springer, Berlin, Germany, 1984.

[5] M. N. Özişik. (1993). Heat Conduction, $2^{\text {nd }}$ edition, Wiley, New York, NY, 1993.

[6] D. Poulikakos. (1993). Conduction Heat Transfer, Prentice Hall, Englewood Cliffs, NJ, 1993.

[7] Y. Yener and S. Kakaç. (2008). Heat Conduction, 4th edition, CRC Press, Boca Raton, FL, 2008.

[8] D. W. Mackowski. (n.d.). Conduction Heat Transfer Notes. http://www.eng.auburn.edu/ dmckwski/mech7210/ condbook.pdf.

[9] http://kitchingroup.cheme.cmu.edu/blog/2013/03/07/Transient-heat-conduction-partial-differential-equations.

[10] O. A. Liskovets. (1965). The method of lines, review (in Russian), Differenzial'nie Uravneniya, Vol. 1, pp. 1662-1668, 1965. English translation: Differential Equations, Vol. 1, pp. 1308-1323, 1965.

[11] E. Isaacson and H. B. Keller. (2012). Analysis of Numerical Methods, Revised edition, Dover Publications, New York, 2012.

[12] G. H. Golub and H. A. van der Vorst. (2000). Eigenvalue computation in the 20th century, Journal of Computational and Applied Mathematics, Vol. 3, No. 1-2, pp. 35-65, 2000.

[13] J. G. Verwer and J. M. Sanz-Serna. (1984). Convergence of method of lines approximations to partial differential equations, Computing, Vol. 33, pp. 297-313, 1984.

[14] S. C. Reddy and L. N. Trefethen. (1992). Stability of the method of lines, Numerische Mathematik, Vol. 62, Issue 1, pp. 235-267, 1992.

[15] A. Zafarullah. (1970). Application of the method of lines to parabolic partial differential equations with error estimates, Journal of the Association for Computing Machinery, Vol. 17, pp. 294-302, 1970.

[16] A. Campo and M. Arıcı. (2019). Semi-analytical, piecewise temperature-time distributions in solid bodies of regular shape affected by uniform surface heat flux: Combination of the Method Of Lines (MOL) and the eigenvalue method, International Communications in Heat and Mass Transfer, Vol. 108, Article \#104276, 2019.

[17] A. Campo. The Numerical Method Of Lines (NMOL) facilitates the instruction of unsteady heat conduction in simple solid bodies with convective surfaces, International Journal of Mechanical Engineering Education. First Published 25 Mar 2020. https://doi.org/10.1177/0306419020910423.

[18] A. Campo and J. Garza. (2014). Transversal Method Of Lines (TMOL) for unsteady heat conduction with uniform surface heat flux, ASME Journal of Heat Transfer, Vol. 136, Paper No. 111302, 2014.

[19] A. Campo and Y. Masip-Macía. (2019). Semi-analytical solution of unsteady heat conduction in a large plane wall with convective boundary conditions for the "small-time" sub-domain using the Transversal Method Of Lines (TMOL), International Journal of Numerical Methods in Heat and Fluid Flow, Vol. 29, Issue 2, pp. 536-552, 2019.

[20] A. Campo and J. Sieres. (2020). Semi-analytical treatment of the unsteady heat conduction equation with prescribed surface temperature: The Transversal Method Of Lines (TMOL) delimited to the "small time" sub-domain, International Communications in Heat and Mass Transfer, Vol. 116, July 2020. Article number 1046872020.

[21] A. Campo and D. J. Celentano. (2020). Improved Transversal Method Of Lines (ITMOL) for unidirectional, unsteady heat conduction in regular solid bodies with heat convection exchange to nearby fluids, Computational Thermal Sciences: An International Journal, Vol. 12, Issue 2, pp. 179-189, 2020. 


\section{Nomenclature}

$c_{v} \quad$ specific heat capacity at constant volume [J/kg K]

$h_{1} \quad$ convection coefficient of hot fluid $1\left[\mathrm{~W} / \mathrm{m}^{2} \mathrm{~K}\right]$

$h_{2} \quad$ convection coefficient of cold fluid $2\left[\mathrm{~W} / \mathrm{m}^{2} \mathrm{~K}\right]$

$k \quad$ thermal conductivity of solid [W/m K]

$L \quad$ thickness of slab [m]

$m \quad$ mass [kg]

$Q \quad$ total heat transfer [W]

$t \quad$ time [s]

$T \quad$ temperature [K]

$T_{\text {in }} \quad$ initial temperature [K]

$T_{f 1} \quad$ temperature of hot fluid $1[\mathrm{~K}]$

$T_{f 2} \quad$ temperature of cold fluid $2[\mathrm{~K}]$

$x \quad$ space coordinate [m]

$X \quad$ dimensionless space coordinate, $\frac{x}{L}$

\section{Greek symbols}

$\alpha \quad$ thermal diffusivity, $\frac{k}{\rho c_{v}}\left[\mathrm{~m}^{2} / \mathrm{s}\right]$

$\Delta x \quad$ spatial interval [m]

$\theta \quad$ dimensionless temperature, $\frac{T-T_{f 2}}{T_{f 1}-T_{f 2}}$

$\rho \quad$ density $\left[\mathrm{kg} / \mathrm{m}^{3}\right]$

$\tau \quad$ dimensionless time, $\frac{t}{L^{2} / \alpha}$

\section{Subscripts}

ls left surface of slab

rs right surface of slab

\section{Appendix: Exact Temperature Field}

The dimensionless exact temperature field in a slab with unequal temperature boundary conditions available in Özişik [5] is

$$
\theta(X, \tau)=X+\frac{2}{\pi} \sum_{n=1}^{\infty} \frac{(-1)^{n}}{n} \sin (n \pi X) e^{-n^{2} \pi^{2} \tau}
$$

The dimensionless exact mean temperature field is defined by

$$
\theta_{m}(\tau)=\int_{0}^{1} \theta(X, \tau) d X
$$

where

$$
\theta_{m}=\frac{T_{m}(t)-T_{f 2}}{T_{f 1}-T_{f 2}}
$$

comes from eq. (42).

Introducing eq. (A.1) into eq. (A.2) and later performing the integration gives at the end

$$
\theta_{m}(\tau)=\frac{1}{2}-\frac{2}{\pi^{2}} \sum_{n=1}^{\infty}\left[\frac{(-1)^{n}+(-1)^{2 n+1}}{n^{2}}\right] e^{-n^{2} \pi^{2} \tau}
$$

